\title{
Evidence for Chirped Auger-Electron Emission
}

\author{
B. Schütte, ${ }^{1,3}$ S. Bauch, ${ }^{2}$ U. Frühling, ${ }^{1}$ M. Wieland,${ }^{1}$ M. Gensch,,${ }^{4,5}$ E. Plönjes,${ }^{4}$ T. Gaumnitz, ${ }^{1}$ A. Azima, ${ }^{1}$ \\ M. Bonitz, ${ }^{2}$ and M. Drescher ${ }^{1, *}$ \\ ${ }^{1}$ Institut für Experimentalphysik, Universität Hamburg, Luruper Chaussee 149, 22761 Hamburg, Germany \\ ${ }^{2}$ Institut für Theoretische Physik und Astrophysik, Christian-Albrechts-Universität, Leibnizstraße 15, 24098 Kiel, Germany \\ ${ }_{3}^{3}$ Max-Born-Institut, Max-Born-Straße 2A, 12489 Berlin, Germany \\ ${ }^{4}$ Deutsches Elektronen-Synchrotron DESY, Notkestraße 85, 22603 Hamburg, Germany \\ ${ }^{5}$ Helmholtz-Zentrum Dresden Rossendorf, Bautzner Landstraße 400, 01328 Dresden, Germany
}

(Received 9 December 2011; published 19 June 2012)

\begin{abstract}
Auger decay carries valuable information about the electronic structure and dynamics of atoms, molecules, and solids. Here we furnish evidence that under certain conditions Auger electrons are subject to an energetic chirp. The effect is disclosed in time-resolved streaking experiments on the $\mathrm{Xe} N O O$ and $\mathrm{Kr}$ $M N N$ Auger decay using extreme-ultraviolet pulses from the free-electron laser in Hamburg as well as from a high-order harmonic laser source. The origin of this effect is found to be an exchange of energy between the Auger electron and an earlier emitted correlated photoelectron. The observed time-dependent spectral modulations are understood within an analytical model and confirmed by extensive computer simulations.
\end{abstract}

DOI: 10.1103/PhysRevLett.108.253003

PACS numbers: 32.80.Aa, 32.80.Hd

The Auger effect [1], i.e., the emission of a secondary electron after a nonradiative decay of a deeply bound electronic core-hole, has found prevalent use as a spectroscopic tool in modern analytical instruments. It derives its capabilities from the fact that the electrons' kinetic energy is an intrinsic property of the electronic structure of the excited atom and does not rely on the energy of the exciting photon, electron, or ion. The electron emission is not monoenergetic, though; the fast-attosecond to femtosecond-decay times imply corresponding spectral widths in a range of a few tens of $\mathrm{meV}$ to a few $\mathrm{eV}$. This time-energy correspondence suggests a uniform distribution of all energy components over time. In this Letter, we demonstrate that under spectroscopically relevant conditions, this presumption is wrong. Instead, our time-resolved experiments show evidence of an energetic chirp, i.e., a pronounced time-dependent variation of the Auger electrons' kinetic energy. A chirp is well established for photoelectron wave packets that are emitted from atoms directly after absorption of a chirped light wave packet. Since the photo-effect exactly transcribes any chirp of the light to the electron wave, this principle has become the basis for ultrafast light pulse characterization methods that take into account spectral phases [2-4]. For the case of Auger electrons, no such transcription mechanism exists; in this Letter, however, we observed a nonlinear energy chirp, which was independent of a deliberately introduced chirp of the ionizing light pulse. It appears as a consequence of the correlated motion of both photo- and Auger electrons in the Coulomb field of the remaining ion. While the underlying mechanism-also known as "postcollision interaction" (PCI) - has extensively been discussed in the literature [5-10], no attention has so far been paid to the consequence of the effect for the temporal properties of the escaping electron wave packets.
In this Letter, we investigate this temporal energy variation in detail by using a terahertz-field-driven streak camera $[11,12]$, where the ionization takes place in the presence of a strong oscillating electric field. We report significantly modified widths of kinetic energy spectra for opposite observation directions and field gradients which clearly indicate a chirp. The observed spectral modulations are reproduced with semiclassical as well as quantum simulations of the dynamics of two well-timed electrons in the combined field of the remaining ion and a timevarying electric field. It turns out that the time-dependent observation of field-assisted electron-electron $(e-e)$ collision reveals details of the electronic correlation with much higher sensitivity than the mere energy-domain approach. In fact, our analysis shows that a significant contribution to the observed mechanism is due to an $e$-e collision taking place at many atomic radii-much more than typically considered in strong-field rescattering experiments [13-15]. The found effect has severe practical implications for atomic and condensed matter physics [16] and for laserassisted experiments with XUV radiation sources: since the time-energy characteristics of Auger-electron emission are used as a probe for inner-shell electron dynamics $[17,18]$, and as a tool for diagnostics of ionizing light pulses $[19,20]$, the found effect has to be taken into account in cases where the photoelectron carries only a small excess kinetic energy.

Recall the mechanism of the PCI effect; cf. Fig. 1: A fast Auger electron (AE) overtakes a slower photoelectron (PE) which causes a temporal change of the effective electronion interaction potential $V_{\text {ei }}$ the two electrons experience. As a consequence, after passing by the PE at a distance $r^{*}$ from the ion, the AE gains (the $\mathrm{PE}$ loses) the energy $\Delta E_{\mathrm{PCI}}=-\frac{1}{r^{*}}-\left(-\frac{2}{r^{*}}\right)=\frac{1}{r^{*}}>0$. Here the main 


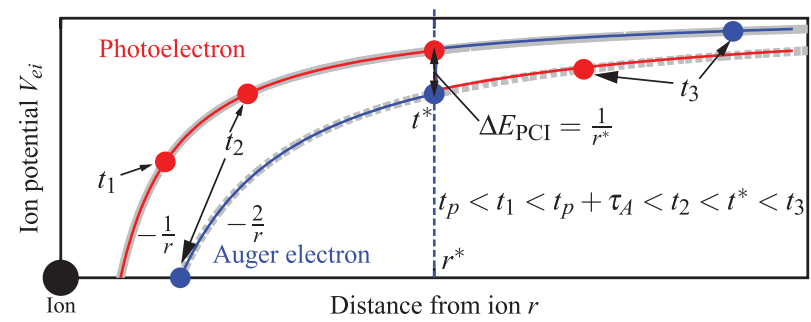

FIG. 1 (color). Scheme of the PCI effect. A photoelectron (PE, created at time $t_{p}$ ) is overtaken by a faster Auger electron (AE, created at time $t_{p}+\tau_{A}$ ) at time $t^{*}$ and distance $r^{*}$ from the ion. The binding potential $V_{\mathrm{ei}}(r)$ changes from $-1 / r$ to $-2 / r$, for the $\mathrm{PE}$, and vice versa for the $\mathrm{AE}$, resulting in an energy gain (loss) $\Delta E_{\mathrm{PCI}}$ of the $\mathrm{AE}(\mathrm{PE})$.

assumptions are that the electrons may be treated classically and that their energy exchange occurs instantaneously, at time moment $t^{*}$ [8]. It is obvious-and will be derived analytically below-that the amount of energy exchange must depend on the relative timing between both electrons. In the following, we extend this model to the presence of an oscillating electric field and find the dependence of $\Delta E_{\mathrm{PCI}}$ on the phase of this field at the instant of PE emission.

Consider an AE emitted with momentum $p_{A}$ at time $\tau_{A}$ after the emission time $t_{p}$ of the PE (having the initial momentum $p_{p}$ ) in the presence of a streaking electromagnetic field with vector potential $A\left(t_{p}+\tau_{A}\right)$. Upon arrival at a remote detector, it has gained an energy

$$
\epsilon\left(\tau_{A}, t_{p}\right)=\frac{1}{2}\left[p_{A}-A\left(t_{p}+\tau_{A}\right)\right]^{2}+\Delta E_{\mathrm{PCI}}-\frac{1}{2} p_{A}^{2} .
$$

In the vicinity of a zero-transition $A\left(t_{p}\right)=0$, the sinusoidal vector potential varies almost linearly, and we can transform Eq. (1) to

$$
\epsilon\left(\tau_{A}, t_{p}\right) \approx-p_{A} \dot{A}_{p} \tau_{A}+\Delta E_{\mathrm{PCI}}+O\left(\tilde{\tau}_{A}^{2}\right),
$$

where we defined $\dot{A}_{p}=\left.d A(t)\right|_{t=t_{p}} / d t$, and terms quadratic in $A$ and in $\tilde{\tau}_{A}$ (the ratio of $\tau_{A}$ to the laser period $T_{L}$ ) have been neglected. Using Eq. (2), the temporal distribution of the AE yield, $T_{A}\left(\tau_{A}\right)=\Gamma_{A} e^{-\Gamma_{A} \tau_{A}}$, where $\Gamma_{A}^{-1}$ denotes the core hole lifetime, can be directly mapped onto an energy distribution $f(\epsilon)$ at the detector. This concept represents a special implementation of a light-driven streak camera $[11,21]$, where temporal information is linearly transferred to an easily measurable energy spectrum. If PCI effects are neglected in Eq. (2), the resulting $A E$ spectrum is exponential as well,

$$
f(\epsilon)=\Gamma_{1} e^{-\Gamma_{1}|\epsilon|}, \quad \Gamma_{1}=\Gamma_{A} /\left(p_{A}\left|\dot{A}_{p}\right|\right),
$$

and exhibits the same width, but an opposite energy gradient, for the rising $(\epsilon<0)$ and falling $(\epsilon>0)$ slope of the vector potential, respectively (see gray curves in Fig. 2).

We now restore the term $\Delta E_{\mathrm{PCI}}$ in Eq. (2). A straightforward calculation yields

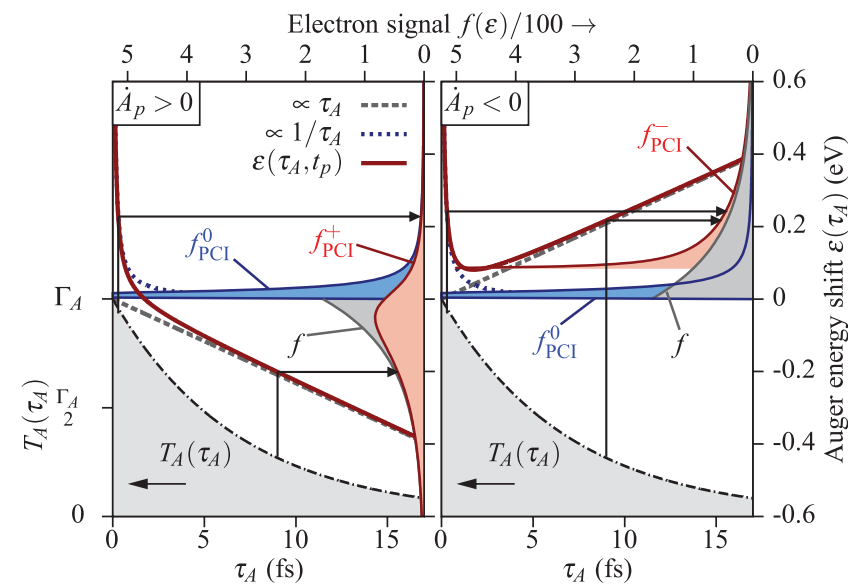

FIG. 2 (color). Light-field-assisted PCI for two successive zeroes of the streaking vector potential, corresponding to positive (left graph) and negative (right graph) time derivative. An AE created at time $\tau_{A}$ after the PE arrives at the detector with an energy gain $\epsilon\left(\tau_{A}, t_{p}\right)$, bold red curve. The exponential ionization probability $T_{A}\left(\tau_{A}\right)$ is mapped onto the AE energy distribution $f_{\mathrm{PCI}}^{ \pm}(\epsilon)$, red curve via Eq. (6), as indicated by the arrows for two Auger release times ( 0.3 and $9 \mathrm{fs})$. In the absence of PCI, the energy distributions in the two cases have the same shape [gray curves labeled $f$, Eq. (3)], deriving from the linear time-energy relation (dashed gray lines). PCI without streaking field causes a broadening of the line and shift to higher energies (blue curves $\left.f_{\mathrm{PCI}}^{0}\right)$. Values refer to Xe NOO Auger decay and streaking with $1 \mathrm{THz}$ at experimental conditions.

$$
r^{*}\left(\tau_{A}, t_{p}\right)=\tau_{A} p_{r}\left(\tau_{A}, t_{p}\right)+O\left[\tilde{\tau}_{A}^{2}\right]
$$

with

$$
p_{r}=\frac{p_{p} p_{A}}{p_{A}-A\left(t_{p}+\tau_{A}\right)-p_{p}+A\left(t_{p}\right)} .
$$

If the streaking field is absent, the PCI effect yields, via Eq. (2), a time-dependent energy shift of $\left.\epsilon\left(\tau_{A}\right)\right|_{A=0}=$ $\Delta E_{\mathrm{PCI}}=\frac{1}{r^{*}}=\frac{1}{\tau_{A} p_{r}^{0}}$, that is mapped onto an energy distribution $f_{\mathrm{PCI}}^{0}(\epsilon)=\frac{\Gamma_{2}}{\epsilon^{2}} e^{-\Gamma_{2} / \epsilon}$, where $\Gamma_{2}=\Gamma_{A} / p_{r}^{0}$ [8] and $p_{r}^{0}=\left.p_{r}\right|_{A=0}$. This spectrum, displayed by the blue curve in Fig. 2, confirms that PCI shifts the Auger spectrum towards higher energies.

Finally, we consider the combined effect of streaking and PCI on the time-energy correspondence of the AE. For PE emission times where $A=0$, Eq. (2) with Eqs. (4) and (5) leads to an energy gain of

$$
\epsilon\left(\tau_{A}, t_{p}\right) \approx-p_{A} \dot{A}_{p} \tau_{A}+\frac{1}{\tau_{A} p_{r}\left(\tau_{A}, t_{p}\right)},
$$

that is displayed in Fig. 2 by the bold red line. For the rising and falling slope of $A(t)$ (left and right diagram, respectively) strikingly different mappings arise: while in the first case the energy shifts extend over the entire energy axis and include negative values caused by the electron deceleration in the streaking field, in the latter case, only 
positive energy shifts that exceed a threshold, $\epsilon_{0}=$ $2\left[p_{A}\left|\dot{A}_{p}\right| / p_{r}\right]^{1 / 2}$, are realized. This indicates that PCI has a nontrivial and rather unexpected effect: in the former case, it acts towards broadening of the energy spectrum whereas, in the latter case, it gives rise to a compression of the line, which clearly indicates a nonlinear energetic chirp. This is readily verified by computing, as before, the energy distribution from the time distribution $T_{A}\left(\tau_{A}\right)$, but now using the full mapping (6). A straightforward calculation yields, for the case $\dot{A}_{p}>0$ :

$$
f_{\mathrm{PCI}}^{+}(\epsilon)=2 \Gamma_{1} \frac{k_{+}-\epsilon}{k_{+}} e^{2 \Gamma_{1}\left[\epsilon-k_{+}\right]},
$$

and, for $\dot{A}_{p}<0$ :

$$
f_{\mathrm{PCI}}^{-}(\epsilon)=4 \Gamma_{1} e^{-2 \Gamma_{1} \epsilon}\left[\frac{\epsilon}{k_{-}} \cosh \left(2 \Gamma_{1} k_{-}\right)-\sinh \left(2 \Gamma_{1} k_{-}\right)\right],
$$

where $k_{ \pm}=\left[\epsilon^{2} \pm \epsilon_{0}^{2}\right]^{1 / 2}$. The two distributions $f_{\mathrm{PCI}}^{ \pm}$are shown in Fig. 2 by the red curves. It is evident that the Auger spectrum $f_{\mathrm{PCI}}^{+}$is substantially broader than $f_{\mathrm{PCI}}^{-}$, in agreement with the above discussion. From our analysis it is obvious that the different widths of the AE spectra at two successive zero crossings of $A$ are a consequence of field-assisted postcollision interaction (FAPCI), i.e., $e-e$ correlations in the combined field of the ion and the electromagnetic wave. We underline that this result holds for arbitrary initial electron momenta as long as $p_{A}$ exceeds $p_{p}$. The strongest FAPCI is expected when $p_{p}$ is small since this reduces $r^{*}$ and, hence, increases $\Delta E_{\mathrm{PCI}}$.

For the experimental investigation of the Auger decay in a light field, terahertz $(\mathrm{THz})$ streaking $[11,12]$ has been implemented, thus ensuring an oscillation period being much longer than the Auger decay time. The effect was investigated in experiments employing two conceptually very different light sources. In the first experiment, $13.5 \mathrm{~nm}$ $(92 \mathrm{eV}) \mathrm{XUV}$ pulses from the free-electron laser in Hamburg (FLASH) [22] were superimposed with intense $92 \mu \mathrm{m}(\approx 3 \mathrm{THz})$ pulses from an in-line THz-undulator [23]. In the second experiment, $13.6 \mathrm{~nm}(91 \mathrm{eV}) \mathrm{XUV}$ pulses from a high-order harmonic generation (HHG) source were combined with single-cycle $\mathrm{THz}$ pulses from an optical rectification source [12,24]. For details of the setups see $[11,12]$. Figure 3 shows series of Xe electron kinetic energy spectra taken with a time-of-flight spectrometer in the presence of a strong THz field at FLASH. Varying the time delay between the pulses reveals the timedependent $\mathrm{THz}$ vector potential, which is proportional to minus the measured energy shift. One can clearly discern an asymmetry in the linewidths at the ascending and the descending slopes of the kinetic energy shift (indicated by white arrows in Fig. 3) for both the $4 d \mathrm{PE}$ and the NOO AE lines [25]. Regarding the PE, this behavior could be interpreted as a result of a negatively chirped XUV pulse $[11,12]$, but for the AE this interpretation must fail. For a quantitative comparison of our experimental results with

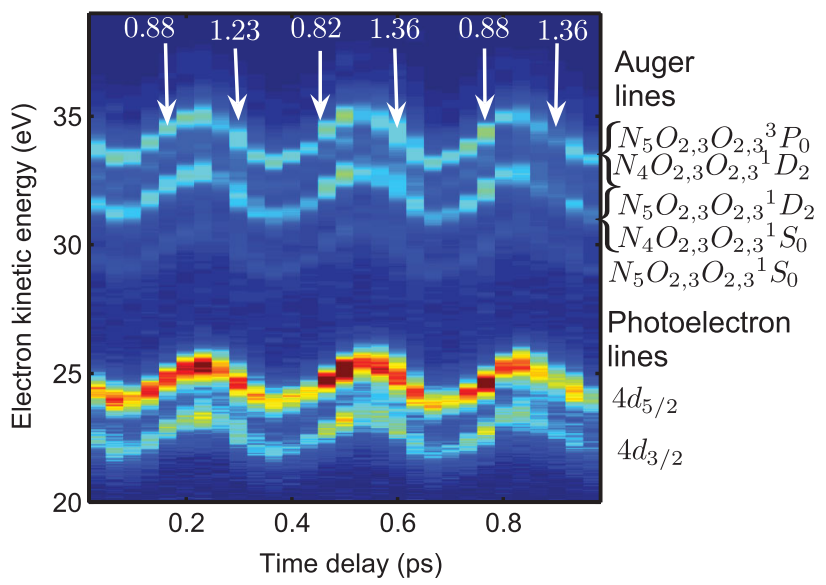

FIG. 3 (color). Series of kinetic energy spectra of Xe $4 d$ photoelectrons and Auger electrons formed after absorption of XUV photons in the presence of a terahertz field with a variable time delay. Data are recorded with the accelerator-based terahertz-field-driven streak camera at field strengths of $120 \mathrm{kV} / \mathrm{cm}$. Note that the ascending (descending) slope of the kinetic energy shift corresponds to the descending (ascending) slope of the streaking vector potential. Marked in white are the FWHM widths of Gaussian functions fitted to the Auger lines of the spectra indicated by the white arrows. The corresponding width uncertainties are $0.03 \mathrm{eV}$.

an interpretation in terms of the above introduced PCIinduced energy chirp, we focus on the measurements performed with the laboratory $\mathrm{HHG}+\mathrm{THz}$ source, because the fluctuating temporal profile and the complex pulse structure at FLASH [11,22] complicate the analysis. However, we underline that the qualitative response of the Auger spectra on the $\mathrm{THz}$ field is very similar for both types of light sources. Xe NOO Auger spectra were measured in three different configurations using $20 \mathrm{fs}$ XUV pulses (Fig. 4). In the field-free case, the spectrum consists of contributions from nine different, partially overlapping Auger lines (cf. [26]) with their individual widths being dominated by the detector resolution of typically $0.5 \mathrm{eV}$ as compared to the natural linewidth of $0.1 \mathrm{eV}$. In order to investigate the influence of PCI on THz streaking, Auger spectra were additionally taken close to a zero-crossing of the kinetic energy shift. The electron kinetic energy analysis with two time-of-flight detectors oriented in opposite directions along the THz and XUV polarization (see [12]) makes it possible to simultaneously record spectra at the ascending and at the descending slope of the kinetic energy shift, thus guaranteeing exactly identical streaking fields for both cases.

For a quantitative comparison with the experiments, the above simple one-dimensional model of FAPCI has to be extended to account for the experimental XUV pulse durations and line shapes as well as for the angular distributions of the (undisturbed) $\mathrm{AE}$ and PE. To this end, extensive molecular dynamics simulations with proper averaging over the initial momenta $p_{p}$ and $p_{A}$ and release 

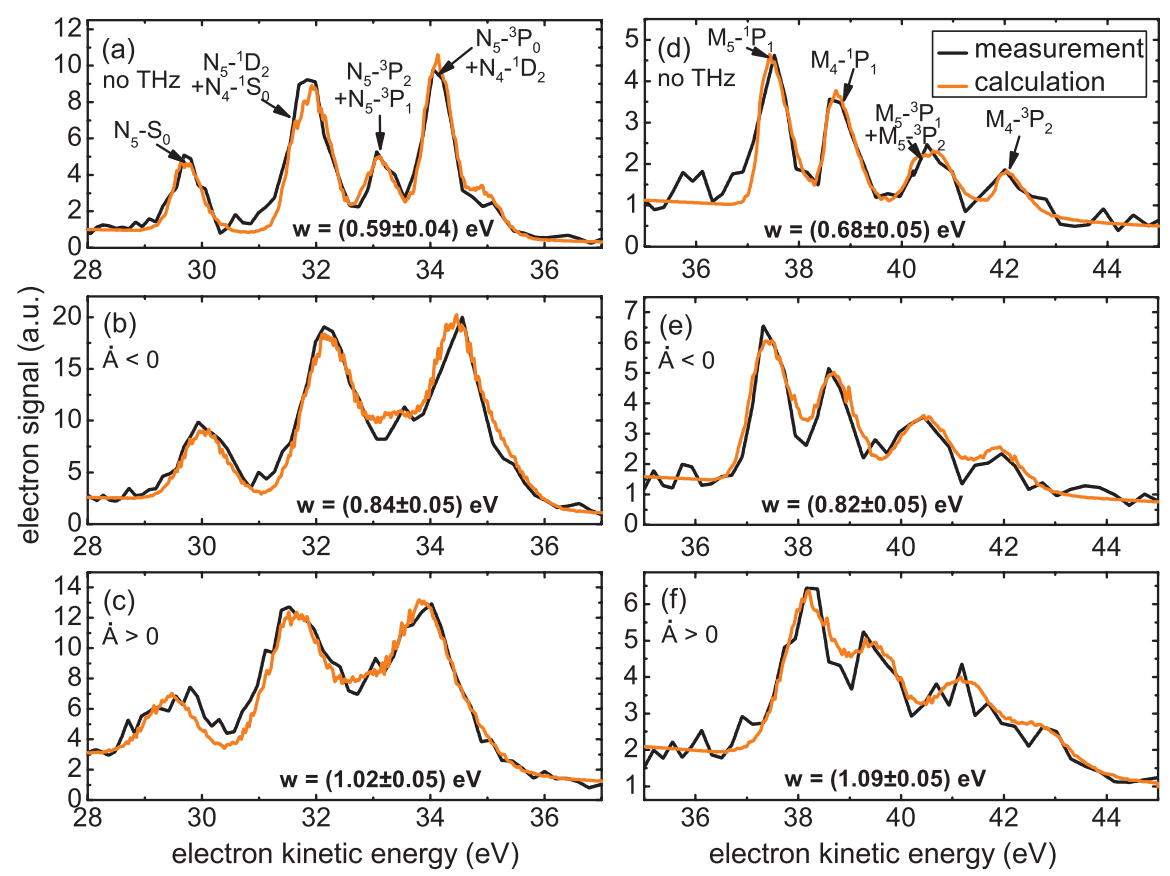

FIG. 4 (color). Comparison of calculated and measured Xe $N O O$ (left) and $\mathrm{Kr} M N N$ (right) Auger spectra with and without $(a, d)$ terahertz field. FAPCI causes a linewidth asymmetry for the spectra measured close to a zero crossing at the descending (b, e) and ascending (c, f) slope of the vector potential. In the Auger line labels, the medium terms $O_{2,3} O_{2,3}$ for $\mathrm{Xe}$ and $N_{1} N_{2,3}$ for $\mathrm{Kr}$ are omitted for a better readability. The FWHM widths $w$ of Gaussian functions fitted to the Auger lines are given.

times $t_{p}$ and $\tau_{A}$ utilizing Monte Carlo algorithms were performed that take all $e-e$ and $e-i$ interactions fully into account. This semiclassical approach was found to agree well with solutions of the time-dependent Schrödinger equation with both laser fields and PCI included [27] that were carried out as described in Ref. [28]. Next the trajectories of molecular-dynamics-simulated electrons were propagated through a model of the time-of-flight spectrometer using the SIMION8 program [29] in order to take the detector resolution and angular acceptance into account. Each individual Auger line was then represented by one simulated spectrum with the relative amplitude and spectral position adjusted according to highly resolved spectra from [26]. In addition, a wedge-shaped background was added to these data in order to smoothly connect to the experimentally obtained values at the lowest and the highest energy part of the spectrum. The overall amplitude was adjusted for a better comparison of calculated and measured data. Finally, the streaked spectra had to be shifted slightly in energy, since the data were taken not exactly at the zero-crossing of the $\mathrm{THz}$ vector potential.

The comparison of experiment and simulations is shown in Fig. 4 and reveals excellent agreement for xenon in the absence of a streaking field (a). With $\mathrm{THz}$ field included clear evidence of an increased spectral width at the ascending slope of the vector potential (c) compared to the descending slope (b) is observed consistently in the measurements and in the simulations. This strongly supports the interpretation of the results in terms of the FAPCI effect. In addition to the linewidth asymmetry, both experiment and theory reveal an energy shift of the spectrum measured at the ascending slope compared to the spectrum at the descending slope of about $0.1 \pm 0.07 \mathrm{eV}$. For proving that the Auger-electron chirp is not a consequence of the XUV chirp, we have applied positively chirped HHG pulses instead of the negatively chirped pulses in Figs. 3 and 4. While the positive chirp was transferred to the photoelectrons by exhibiting a reversed linewidth asymmetry, the linewidth asymmetry of the Auger electrons remained unaffected. At the used photon energy of $91 \mathrm{eV}$, the $\mathrm{Xe} 4 d$ PE energy is above $20 \mathrm{eV}$, such that the process of the $\mathrm{AE}$ overtaking the $\mathrm{PE}$ occurs relatively far from the ion. In order to further support our interpretation, we create a situation where this process takes place closer to the ion. To this end, $\mathrm{Kr}$ is used as the target medium, and the photon energy is increased to $97.5 \mathrm{eV}$ (right column of Fig. 4). In this case, the kinetic energy of the $3 d_{3 / 2}$ and $3 d_{5 / 2} \mathrm{PE}$ is only $2.5 \mathrm{eV}$ and $3.7 \mathrm{eV}$, respectively, and the AE kinetic energy is additionally increased [cf. Figs. 4(a) and 4(d)], thus drastically increasing $p_{r}$ in comparision to $\mathrm{Xe} N O O$. Well in accordance with our model, the linewidth asymmetry of spectra recorded at the ascending (f) and the descending (e) slope, is now found to be considerably larger than before. Also the energy shift between the spectra measured at the ascending and at the descending slopes is increased to $0.4 \pm 0.15 \mathrm{eV}$ being a further indication for an enhanced influence of FAPCI in the $\mathrm{Kr}$ data (cf. Fig. 2). Given the fact that our detector resolution does 
not allow us to consider an isolated Auger line, the measured spectra are well represented by the simulation results. Residual deviations may result from the uncertainty of the XUV pulse duration measurement as well as from the slightly different natural linewidths of the different Auger lines contained in one spectrum [30].

In summary, our measurements of THz-assisted Auger emission from $\mathrm{Xe}$ and $\mathrm{Kr}$ targets using two completely different XUV or $\mathrm{THz}$ light sources consistently confirm that AEs carry an energy chirp, if their kinetic energy is larger than the one of the primary PE. This general property rests on the correlated motion of both particles in the ion's Coulomb field and their "collision" at time $t^{*}$ at distance $r$ ", at which they exchange an energy $\Delta E_{\mathrm{PCI}}$. The dependence of the Auger-energy shift $\epsilon$ on the emission time imprints a nonlinear (hyperbolic) energy chirp onto the AE wave packet, which is not detectable in pure energy-domain measurements but becomes clearly visible upon streaking at opposite slopes of a $\mathrm{THz}$ vector potential. In spite of an obvious mismatch between the XUV pulse duration (20 fs for the HHG source) and the Auger decay time constant (7.9 fs for $\mathrm{Kr}[31]$ ), the predicted asymmetries are clearly resolved. Moreover, they remain discernible for rather fast $\mathrm{PE}$, where energy-domain measurements do no longer reveal discernible PCI [6]. THz-assisted PCI therefore is able to resolve electronic correlation even at large distances from the ionic core; for the case of Xe, the collision process for an $\mathrm{AE}$ released at, for example, $\tau_{A}=1 \mathrm{fs}$ occurs at a (classical) distance of approximately $20 \mathrm{~nm}$, i.e., 380 atomic radii. We finally note, that besides offering a novel tool for precisely studying details of electron correlation, our finding has immediate consequences for time metrology at pulsed XUV and X-ray sources. Spectral widths of electrons from light-assisted photoemission are evaluated for extracting information on pulse durations and in particular spectral chirps $[3,4,11,12]$. At light sources with large spectral widths of the radiation, such as SASE FELs, the use of AE may result in an improved temporal resolution of the streak camera compared to measurements employing broad PE spectra. Our results indicate, however, that in the discussed regime of kinetic energies, FAPCI introduces significant distortions of spectral shapes, thus potentially leading to erroneous results. Therefore, if Auger decay is involved in such measurements, one either has to properly account for the FAPCI effect or the considered PE should be chosen to be significantly faster than the Auger electrons.

B.S. and S.B. contributed equally to this work. The authors acknowledge discussions with N. Kabachnik on PCI and financial support from the DFG (via Graduiertenkolleg 1355, SFB 925, and project BO13669/1), the Landesexzellenzinitiative Hamburg, the Joachim Herz Stiftung, the BMBF-Verbund "FLASH" and Grant No. shp0006 for computer time at HLRN. *markus.drescher@desy.de

[1] P. Auger, Compt. Rend. 180, 65 (1925).

[2] J. Itatani, F. Quere, G. L. Yudin, M. Y. Ivanov, F. Krausz, and P. B. Corkum, Phys. Rev. Lett. 88, 173903 (2002).

[3] R. Kienberger et al., Nature (London) 427, 817 (2004).

[4] Y. Mairesse and F. Quere, Phys. Rev. A 71, 011401 (2005).

[5] R. B. Barker and H. W. Berry, Phys. Rev. 151, 14 (1966).

[6] V. Schmidt, N. Sandner, W. Mehlhorn, M. Y. Adam, and F. Wuilleumier, Phys. Rev. Lett. 38, 63 (1977).

[7] A. Niehaus, J. Phys. B 10, 1845 (1977).

[8] G. N. Ogurtsov, J. Phys. B 16, L745 (1983).

[9] P. van der Straten, R. Morgenstern, and A. Niehaus, Z. Phys. D 8, 35 (1988).

[10] T. Åberg, Phys. Scr. T41, 71 (1992).

[11] U. Frühling et al., Nature Photon. 3, 523 (2009).

[12] B. Schütte, U. Frühling, M. Wieland, A. Azima, and M. Drescher, Opt. Express 19, 18833 (2011).

[13] M. Meckel et al., Science 320, 1478 (2008).

[14] M. Lein, J. Phys. B 40, R135 (2007).

[15] S. Bauch, K. Balzer, and M. Bonitz, Europhys. Lett. 91, 53001 (2010)

[16] E. E. Krasovskii and M. Bonitz, Phys. Rev. Lett. 99, 247601 (2007).

[17] M. Drescher, M. Hentschel, R. Kienberger, M. Uiberacker, V. Yakovlev, A. Scrinzi, T. Westerwalbesloh, U. Kleineberg, U. Heinzmann, and F. Krausz, Nature (London) 419, 803 (2002).

[18] M. Uiberacker et al., Nature (London) 446, 627 (2007).

[19] J. M. Schins, P. Breger, P. Agostini, R. C. Constantinescu, H. G. Muller, G. Grillon, A. Antonetti, and A. Mysyrowicz, Phys. Rev. Lett. 73, 2180 (1994).

[20] S. Düsterer et al., New J. Phys. 13, 093024 (2011).

[21] M. Hentschel, R. Kienberger, Ch. Spielmann, G. A. Reider, N. Milosevic, T. Brabec, P. Corkum, U. Heinzmann, M. Drescher, and F. Krausz, Nature (London) 414, 509 (2001).

[22] W. Ackermann et al., Nature Photon. 1, 336 (2007).

[23] M. Gensch et al., Infrared Phys. Technol. 51, 423 (2008).

[24] J. Hebling, G. Almási, I.Z. Kozma, and J. Kuhl, Opt. Express 10, 1161 (2002).

[25] Note that the ascending (descending) slope of the kinetic energy shift corresponds to the descending (ascending) slope of the streaking vector potential.

[26] L. O. Werme, T. Bergmark, and K. Siegbahn, Phys. Scr. 6, 141 (1972).

[27] S. Bauch and M. Bonitz, Phys. Rev. A 85, 053416 (2012).

[28] A. K. Kazansky, I. P. Sazhina, and N. M. Kabachnik, J. Phys. B 42, 245601 (2009).

[29] D. A. Dahl, Int. J. Mass Spectrom. 200, 3 (2000).

[30] M. Jurvansuu, A. Kivimaki, and S. Aksela, Phys. Rev. A 64, 012502 (2001).

[31] T. Uphues, M. Schultze, M. F. Kling, M. Uiberacker, S. Hendel, U. Heinzmann, N.M. Kabachnik, and M. Drescher, New J. Phys. 10, 025009 (2008). 